\title{
Results of aortic valve replacement for aortic stenosis in patients aged 80 years and older
}

\author{
Kenji Kuwaki*, Atsushi Amano, Hirotaka Inaba, Taira Yamamoto, Shizuyuki Dohi, \\ Takeshi Matsumura, Satoshi Matsushita
}

Department of Cardiovascular Surgery, Juntendo University, Tokyo, Japan

Email: ${ }^{\text {kuwakikj@yahoo.co.jp }}$

Received 12 May 2012; revised 15 June 2012; accepted 27 June 2012

\begin{abstract}
Purpose: The aim of this study was to investigate our clinical experience in a contemporary series of aortic valve replacement (AVR) for aortic stenosis (AS) with or without coronary artery bypass grafting (CABG), and compare the early and mid-term results between patients aged more than 80 years and those aged less than 80 years. Methods: A retrospective review was performed of 258 consecutive patients with aortic stenosis (AS) who underwent AVR between August 2002 and December 2010 at Juntendo University Hospital. Results: Operative mortality was $7.3 \%$ in patients aged 80 years and older, compared with $3.6 \%$ in younger patients. Thus, operative mortality was higher in the older patients compared with the younger patients, but was not statistically significant between the two groups. Age more than 80 years was not a predictor of operative mortality. Advanced NYHA class $(p=0.03$; odds ratio $[O R], 9.5)$ was found to be a multivariate independent predictor of operative mortality. Patients aged 80 years and older were significantly more likely to suffer from respiratory failure after surgery than younger patients, but there were no significant differences in any other complications between the two age groups. Non-home discharge rate was significantly higher in the patients aged 80 years and older $(21.9 \%)$ than in the younger patients $(5.5 \%)$. Three-year survival was $84 \%$ in patients aged more than 80 years, and $83 \%$ of these elderly patients were living at home at the last follow-up. Conclusions: Patients aged 80 years and older have acceptable results of AVR with slightly increased risk of early mortality and morbidity compared with younger patients.
\end{abstract}

Keywords: Aortic Valve Replacement; Octogenarians

\section{INTRODUCTION}

Life expectancy is steadily increasing in developed coun-

"Corresponding author. tries, and more than 8.4 million people aged 80 years and older live in Japan. In this population, the most common structural cardiac disease is degenerative calcified aortic stenosis [1]. Current guidelines [2,3] demonstrated AVR as class I recommendation in symptomatic patients with AS, however, there remains significant reluctance to recommend AVR in patients greater than 80 years old [4] due mainly to the increased risk of operative mortality and morbidity in this age group. A recent study indicated that about $40 \%$ of octogenarians with AS either refused or were not proposed for AVR, although the operation was indicated on the basis of current guidelines [4].

In recent years, transcatheter aortic valve implantation (TAVI) has emerged as a less invasive alternative to AVR for patients with AS considered to be at very high surgical risk. However, 30-day and 1-year cumulative mortality rates after TAVI have been reported to be $3.4 \%$ to $10 \%$ and $22.1 \%$ to $24.2 \%$, respectively $[5,6]$, and controversy exists about whether high-risk elderly patients actually benefit from TAVI in terms of early and midterm outcomes compared with conventional AVR. Therefore, there is increasing interest in evaluation of outcomes after AVR in elderly patients. The aim of this study was to report our clinical experience in a contemporary series of AVR for AS with or without CABG, compare the early and mid-term results between patients aged more than 80 years and those aged less than 80 years, and determine the risk factors for early morbidity and mortality, non-home discharge, and mid-term mortality.

\section{MATERIALS AND METHODS}

\subsection{Patients and Data}

Between August 2002 and December 2010, 258 consecutive patients underwent AVR for AS at Juntendo University Hospital, including 82 patients with concomitant CABG. Patients were excluded from this cohort study if they required concomitant mitral or tricuspid valve surgery. This study was approved by the Medical 
Ethics Committee of Juntendo University. Baseline preoperative characteristics of this study cohort are listed in Table 1. Outcome measures included operative mortality, postoperative complications (respiratory failure, renal failure, stroke, reoperation for any reason, deep sternal wound infection, gastrointestinal complication, systemic infection, pacemaker implantation for complete atrioventricular heart block), non-home discharge, and mid-term mortality. Operative mortality was defined as death within 30 days of surgery or as death at any time before discharge from hospital. Renal failure was defined as creatinine level greater than $2 \mathrm{mg} / \mathrm{dL}$ postoperatively or new requirement for dialysis postoperatively. Respiratory failure was defined as prolonged postoperative ventilation ( $>24$ hours) or need for reintubation or tracheostomy. Stroke was defined as a new permanent neurological event postoperatively.

\subsection{Operative Procedures}

All surgical procedures were performed through a median sternotomy using cardiopulmonary bypass with systemic normothermia. Myocardial protection was achieved using antegrade and retrograde administration of high-potassium cold blood cardioplegia. Concomitant CABG was performed in 82 patients (Table 2).

\subsection{Statistical Analysis}

Categorical variables are given as percentage and were compared between groups using chi-square test or Fischer's exact test. Continuous variables are reported as mean \pm standard deviation and were compared between groups using the unpaired t-test. Variables that achieved a $\mathrm{p}$ value less than 0.1 in the univariate analysis were then examined using multivariate analysis by multiple logistic regression to evaluate independent risk factors for outcomes. Independent predictors of mid-term survival were determined with the Cox proportional hazards multivariate analysis. A p value less the 0.05 was considered statistically significant. Statistical analyses were performed using SPSS 15 (SPSS, Inc., Chicago, IL). Variables examined by univariate and multivariate analysis included the following: gender, age $\geq 80$ years, left ventricular ejection fraction $(\mathrm{EF}) \leq 40 \%$, body surface area, New York Heart Association (NYHA) class, mean aortic transvalvular pressure gradient $(\mathrm{mpg}) \leq 40 \mathrm{mmHg}$, peak aortic transvalvular pressure gradient (ppg) $\leq 60 \mathrm{mmHg}$,

Table 1. Patients characteristics.

\begin{tabular}{|c|c|c|c|c|c|}
\hline & All patients & Age $<60$ & Age $60-69$ & Age $70-79$ & Age $\geq 80$ \\
\hline & $(\mathrm{n}=258)$ & $(\mathrm{n}=31,12.0 \%)$ & $(\mathrm{n}=75,29.1 \%)$ & $(\mathrm{n}=111,43.0 \%)$ & $(\mathrm{n}=41,15.9 \%)$ \\
\hline Age, mean $\pm \mathrm{SD}$ & $70.3 \pm 9.6$ & $52.3 \pm 7.2$ & $64.7 \pm 2.9$ & $74.7 \pm 2.8$ & $82.5 \pm 2.4$ \\
\hline Male, n (\%) & $145(56)$ & 18 & 45 & 66 & 16 \\
\hline Body surface area, $\mathrm{m}^{2}$, mean & 1.5 & 1.6 & 1.6 & 1.5 & 1.4 \\
\hline Ejection fraction, $\%$, mean & 63 & 64 & 63 & 62 & 64 \\
\hline Ejection fraction $\leq 40 \%, \mathrm{n}(\%)$ & $28(11)$ & 4 & 8 & 10 & 6 \\
\hline NYHA class III-IV, n (\%) & $118(45.7)$ & 7 & 34 & 59 & 19 \\
\hline Dialysis, n (\%) & $27(10.4)$ & 3 & 9 & 14 & 1 \\
\hline Renal failure (creatine $\geq 2.0 \mathrm{mg} / \mathrm{dL}$ or dialysis), $\mathrm{n}(\%)$ & $31(12.1)$ & 3 & 9 & 16 & 3 \\
\hline Atrial fibrillation, n (\%) & $13(5.0)$ & 0 & 3 & 4 & 6 \\
\hline History of stroke, n (\%) & $22(8.5)$ & 3 & 6 & 11 & 2 \\
\hline Peripheral vascular disease, n (\%) & $16(6.2)$ & 1 & 3 & 7 & 5 \\
\hline Bicuspid aortic valve, n (\%) & $50(19.3)$ & 12 & 28 & 10 & 0 \\
\hline History of myocardial infarction, $\mathrm{n}(\%)$ & $22(8.5)$ & 3 & 4 & 11 & 4 \\
\hline COPD, n (\%) & $17(6.6)$ & 2 & 2 & 11 & 2 \\
\hline Hypertension, n (\%) & $190(73.6)$ & 18 & 50 & 86 & 36 \\
\hline Diabetes mellitus, n (\%) & $70(27.1)$ & 7 & 18 & 37 & 8 \\
\hline Smoking (ever), n (\%) & $95(36.8)$ & 13 & 24 & 46 & 12 \\
\hline Resternotomy, n (\%) & $6(2.3)$ & 1 & 1 & 4 & 0 \\
\hline Coronary artery disease, $\mathrm{n}(\%)$ & $82(31.7)$ & 7 & 15 & 46 & 14 \\
\hline Logistoc EuroSCORE, mean $\pm \mathrm{SD}, \%$ & $6.5 \pm 6.6$ & $2.5 \pm 2.3$ & $5.1 \pm 3.9$ & $7.3 \pm 5.8$ & $12.2 \pm 8.5$ \\
\hline Additive EuroSCORE, mean $\pm \mathrm{SD}, \%$ & $6.0 \pm 2.3$ & $3.0 \pm 1.4$ & $4.6 \pm 1.6$ & $6.7 \pm 1.6$ & $8.7 \pm 1.6$ \\
\hline STS-PROM, mean \pm SD, $\%$ & $4.6 \pm 5.8$ & $2.3 \pm 4.3$ & $3.1 \pm 4.8$ & $5.1 \pm 5.3$ & $7.7 \pm 8.1$ \\
\hline
\end{tabular}


Table 2. Operative procedures.

\begin{tabular}{|c|c|c|c|c|c|}
\hline & All patients & Age $<60$ & Age $60-69$ & Age $70-79$ & Age $\geq 80$ \\
\hline & $(\mathrm{n}=258)$ & $(\mathrm{n}=31,12.0 \%)$ & $(\mathrm{n}=75,29.1 \%)$ & $(\mathrm{n}=111,43.0 \%)$ & $(\mathrm{n}=41,15.9 \%)$ \\
\hline \multicolumn{6}{|l|}{ Valve type implanted } \\
\hline Bioprosthetic valve, n (\%) & $183(70.9)$ & $7(22.5)$ & $35(46.6)$ & $100(90.1)$ & $41(100)$ \\
\hline Mechanical valve, n (\%) & $75(29.1)$ & $24(77.4)$ & $40(53.3)$ & $11(9.9)$ & $0(0)$ \\
\hline \multicolumn{6}{|l|}{ Valve size implanted (mm) } \\
\hline$\leq 20 \mathrm{~mm}$ & $72(27.9)$ & $5(16.1)$ & $23(30.1)$ & $24(21.6)$ & $20(48.7)$ \\
\hline $21-22 \mathrm{~mm}$ & $84(32.5)$ & $13(41.9)$ & $21(28.0)$ & $37(33.3)$ & $13(31.7)$ \\
\hline $23-24 \mathrm{~mm}$ & $75(29.1)$ & $9(29.0)$ & $23(30.1)$ & $35(31.5)$ & $8(19.5)$ \\
\hline$\geq 25 \mathrm{~mm}$ & $27(10.4)$ & $4(12.9)$ & $8(10.6)$ & $15(13.5)$ & $0(0)$ \\
\hline Concomitant CABG, n (\%) & $82(31.7)$ & $7(22.5)$ & $15(20.0)$ & $46(41.4)$ & $14(34.1)$ \\
\hline Aortic cross-clamp time, minutues, mean $\pm \mathrm{SD}$ & $112 \pm 35$ & $114 \pm 35$ & $113 \pm 42$ & $112 \pm 31$ & $110 \pm 35$ \\
\hline Cardiopulmonary bypass time, minutues, mean \pm SD & $148 \pm 52$ & $144 \pm 47$ & $156 \pm 71$ & $146 \pm 39$ & $143 \pm 42$ \\
\hline \multicolumn{6}{|l|}{ No. of coronary artery bypass grafts } \\
\hline mean & 2.3 & 2.3 & 2.4 & 2.2 & 2.7 \\
\hline 1-graft, n (\%) & $27(10.4)$ & $3(9.6)$ & $4(5.3)$ & $17(15.3)$ & $3(7.3)$ \\
\hline 2-grafts, n (\%) & $23(8.9)$ & $1(3.2)$ & $5(6.6)$ & $14(12.6)$ & $3(7.3)$ \\
\hline 3-grafts, n (\%) & $15(5.8)$ & $2(6.4)$ & $2(2.6)$ & $7(6.3)$ & $4(9.7)$ \\
\hline$\geq 4$-grafts, $\mathrm{n}(\%)$ & $17(6.6)$ & $1(3.2)$ & $4(5.3)$ & $8(7.2)$ & $4(9.7)$ \\
\hline
\end{tabular}

creatinine $\geq 2 \mathrm{mg} / \mathrm{dL}$, atrial fibrillation, coronary artery disease requiring $\mathrm{CABG}$, valve prosthesis type, peripheral vascular disease, diabetes mellitus, hyperten- sion, smoking, old myocardial infarction, and chronic obstructive pulmonary disease (COPD).

\section{RESULTS}

Demographic Data, Preoperative Risk Factors, and Operative Data.

A total of 258 patients were studied. The preoperative patient characteristics are listed in Table 1. Table 2 represents the operative characteristics.

\subsection{Operative Mortality}

Operative mortality in patients aged more than 80 years and younger patients was $7.3 \%(n=3)$ and $3.6 \%(n=8)$, respectively (Table 3). Operative mortality was higher in the older patients compared with the younger patients, but was not statistically significant. On univariate analysis, EF $\leq 40 \%$, NYHA class III-IV, creatinine $\geq 2 \mathrm{mg} / \mathrm{dL}$, diabetes mellitus, and old myocardial infarction emerged as risk factors of operative mortality. On multivariate analysis, NYHA class III-IV ( $p=0.03 ; 95 \%$ confidence interval [CI], 1.13 to 81.30 , odds ratio [OR], 9.5; Table 4) was identified as an independent variable of operative mortality for the overall population. Operative mortalities were $0.1 \%(\mathrm{n}=1)$ and $8.4 \%(\mathrm{n}=10)$ for patients with NYHA class II $(\mathrm{n}=139)$ and NYHA class III-IV (n $=119)$, respectively $(\mathrm{p}=0.002)$.

\subsection{Postoperative Complications}

Forty-six patients $(17.8 \%)$ developed at least one postoperative complication (Table 3). Postoperative complication occurred in 12 patients $(29.2 \%)$ aged more than 80 years and 29 patients $(13.3 \%)$ in the younger patients ( $p$ $=0.03)$. Multivariate analysis identified NYHA class III-IV ( $\mathrm{p}=0.02 ; 95 \% \mathrm{CI}, 1.13$ to 5.52, OR, 2.4; Table 4) and age $\geq 80$ years $(\mathrm{p}=0.02 ; 95 \% \mathrm{CI}, 1.16$ to 7.33 , OR, 2.9 ) as independent predictors of postoperative complication. Among the major postoperative complications examined, the incidence of respiratory failure was significantly greater in the patients aged 80 years and older, but there were no significant differences in any other complications between the age groups (Table 3). Therefore, the higher incidence of postoperative complications in patients aged 80 years and older is explained by a greater incidence of respiratory failure in this group. Size and type of the valve implanted had no effect on early mortality and morbidity in this study.

\subsection{Discharge to Non-Home Location}

Non-home discharge rate was significantly higher in the patients aged 80 years and older $(n=9,21.9 \%)$ than in the younger patients $(n=12,5.5 \%)(p=0.001)$. Multivariate analysis revealed that age $\geq 80$ years $(\mathrm{p}=0.002$; 95\% CI, 2.04 to 25.78 , OR, 7.2), NYHA class III-IV ( $\mathrm{p}=$ $0.01 ; 95 \% \mathrm{CI}, 1.37$ to $17.63, \mathrm{OR}, 4.9)$, and creatinine $\geq 2$ $\mathrm{mg} / \mathrm{dL}(\mathrm{p}=0.03 ; 95 \% \mathrm{CI}, 1.07$ to 18.75 , OR, 4.4) were significantly associated with non-home discharge (Table 4). 
Table 3. Operative outcomes.

\begin{tabular}{|c|c|c|c|c|c|}
\hline & All patients & Age $<60$ & Age $60-69$ & Age $70-79$ & Age $\geq 80$ \\
\hline & $(\mathrm{n}=258)$ & $(\mathrm{n}=31,12.0 \%)$ & $(\mathrm{n}=75,29.1 \%)$ & $(\mathrm{n}=111,43.0 \%)$ & $(\mathrm{n}=41,15.9 \%)$ \\
\hline Operative mortality, n (\%) & $11(4.2)$ & $0(0)$ & $3(4.0)$ & $5(4.5)$ & $3(7.3)$ \\
\hline Postoperative any complication, $\mathrm{n}(\%)$ & $46(17.8)$ & $3(9.6)$ & $8(10.6)$ & $23(20.7)$ & $12(29.2)$ \\
\hline Permanent stroke, n (\%) & $6(2.3)$ & $0(0)$ & $2(2.6)$ & $3(2.7)$ & $1(2.4)$ \\
\hline Renal failure, n (\%) & $16(6.2)$ & $0(0)$ & $5(6.6)$ & $9(8.1)$ & $2(4.8)$ \\
\hline Respiratory failure, n (\%) & $30(11.6)$ & $2(6.4)$ & $4(5.3)$ & $14(12.6)$ & $10(24.3)$ \\
\hline Deep strernal wound infection, $\mathrm{n}(\%)$ & $0(0)$ & $0(0)$ & $0(0)$ & $0(0)$ & $0(0)$ \\
\hline Reoperation for any reason, $\mathrm{n}(\%)$ & $5(1.9)$ & $0(0)$ & $1(1.3)$ & $3(2.7)$ & $1(2.4)$ \\
\hline Gastrointestinal complications, $\mathrm{n}(\%)$ & $6(2.3)$ & $0(0)$ & $1(1.3)$ & $5(4.5)$ & $0(0)$ \\
\hline Systemic infection, n (\%) & $4(1.5)$ & $0(0)$ & $1(1.3)$ & $3(2.7)$ & $0(0)$ \\
\hline Prolonged length of stay $>14$ days, $n(\%)$ & $63(24.4)$ & $10(32.2)$ & $14(18.6)$ & $28(25.2)$ & $11(25.8)$ \\
\hline Non-home discharge, n (\%) & $21(8.5)$ & $1(3.2)$ & $3(4.0)$ & $8(7.2)$ & $9(23.6)$ \\
\hline
\end{tabular}

Table 4. Independent predictors of early and mid-term outcomes.

\begin{tabular}{llll}
\hline \multicolumn{1}{c}{ Variable } & Odds Ratio & p Value \\
\hline Operative mortality & & & \\
NYHA class III-IV & 9.5 & $1.13-81.30$ & 0.03 \\
Postoperative complication & & & 0.02 \\
NYHA class III-IV & 2.4 & $1.13-5.52$ & 0.02 \\
Age $\geq 80$ years & 2.9 & $1.16-7.33$ & 0.002 \\
Non-home discharge & & & 0.01 \\
Age $\geq 80$ years & 7.2 & $2.04-25.78$ & 0.03 \\
NYHA class III-IV & 4.9 & $1.37-17.63$ & $1.07-18.75$ \\
Creatinine $\geq 2$ mg/dL & 4.4 & & 0.0004 \\
Mid-term mortality & & $3.20-17.82$ & 0.008 \\
Creatinine $\geq 2$ mg/dL & 4.8 & $2.64-48.64$ & 0.04 \\
NYHA class III-IV & 7.3 & $1.24-11.03$ & \\
Peripheral vascular disease & 3.1 & & \\
\hline
\end{tabular}

\subsection{Mid-Term Survival}

Survival estimates including operative mortality, at 1, 3, and 5 years were $89.8 \%, 83.8 \%$, and $83.8 \%$ for the patients aged more than 80 years versus $94.4 \%, 92.6 \%$, and $89.3 \%$ for the younger age patients (Figure 1). There was no statistical significance between the two age groups regarding mid-term survival. Multivariate analysis identified NYHA class III-IV ( $\mathrm{p}=0.008 ; 95 \% \mathrm{CI}$, 2.64 to 48.64 , OR, 7.3), creatinine $\geq 2 \mathrm{mg} / \mathrm{dL}(\mathrm{p}=$ $0.0004 ; 95 \% \mathrm{CI}, 3.20$ to 17.82 , OR, 4.8), and peripheral vascular disease $(\mathrm{p}=0.04 ; 95 \% \mathrm{CI}, 1.24$ to $11.03, \mathrm{OR}$, 3.1) as independent predictors of mid-term mortality (Table 4). The 5-year survival of patients undergoing surgery in NYHA class III-IV (79.3\%) was significantly lower than that of patients in NYHA class II $(98.5 \%)$ (log-rank, $\mathrm{p}<0.001 ;$ Figure 2).

\section{DISCUSSION}

With increasing number of patients with AS, particularly in elderly patients with relevant concomitant disease, being referred for surgery, evaluation of surgical results in patients aged more than 80 years has become a major concern $[1,4]$. In recent years, TAVI has emerged as a less invasive alternative to AVR for elderly patients with AS at very high surgical risk, particularly in octogenarians, but controversy exists about whether high risk elderly patients actually benefit from TAVI compared with AVR [6]. Therefore, we have evaluated the early and mid-term outcomes after AVR between patients aged more than 80 years and those aged less than 80 years, 


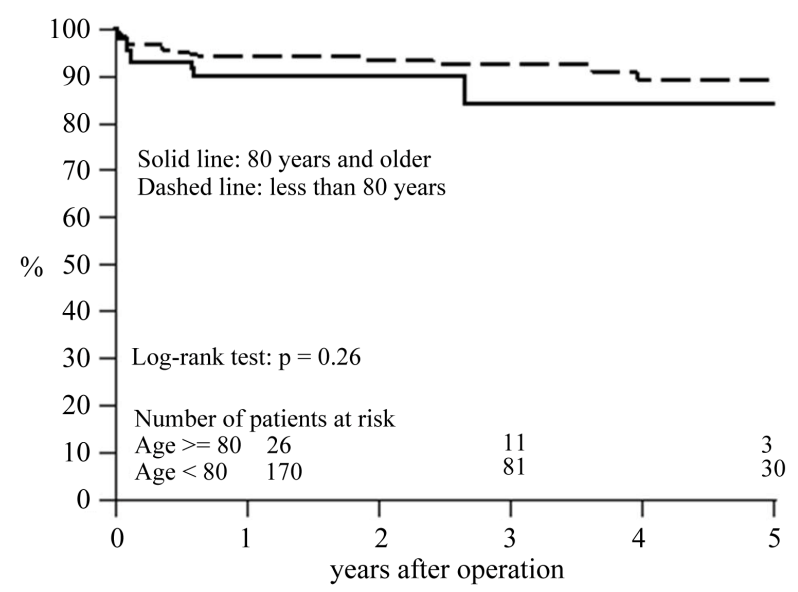

Figure 1. Overall survival stratified by age: 80 years and older (solid line) and less than 80 years (dashed line). No statistically significant difference $(83 \%$ versus $89 \%$ at 5 years; $p=0.26$ ) was seen between the two age groups.

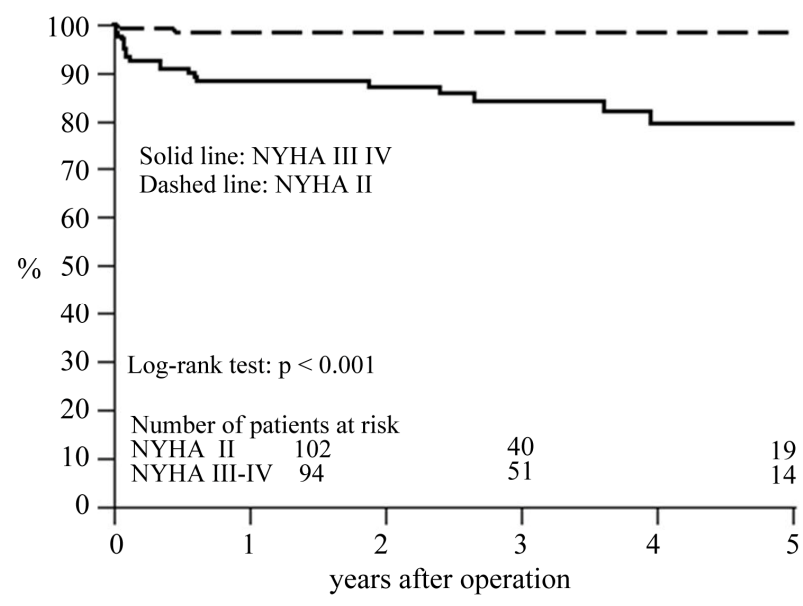

Figure 2. Overall survival stratified by NYHA class: NYHA class III-IV (solid line) and NYHA class II (dashed line). A statistically significant difference $(79 \%$ versus $98 \%$ at 5 years; $\mathrm{p}<0.001)$ was seen between the two groups.

and determine the risk factors for early morbidity and mortality, non-home discharge, and mid-term mortality.

\subsection{Operative Mortality}

The results of the current study demonstrate that good results after AVR can be expected in patients aged 80 years and older with slightly increased risk of operative mortality and morbidity compared with younger patients. Mid-term results in patients aged 80 years and older are also satisfactory with more than $80 \%$ of survival at 3 years. Previous studies, in the mid-1990s and earlier, analyzing results of isolated AVR or AVR + CABG reported operative mortality between $9 \%$ and $28 \%$ in patients aged over 80 years [7-9]. However, studies in the last decade have demonstrated improvement in operative mortality between 5\% and 10\% [10-15] (Table 5).
Therefore, the operative mortality of $7.3 \%$ in the current study is consistent with recent reports of large series for octogenarians.

Improvement of operative outcome after AVR in recent years may be related to advances in perioperative management and continued refinements including surgical technique, cardiac anesthesia, meticulous myocardial protection, and postoperative management and rehabilitation. Our routine adjunctive perioperative practice includes CT scan for detection of vascular calcification, preoperative nasal culture of MRSA (methicillin-resistant staphylococcus aureus), both antegrade and retrograde administration of cardioplegia, and routine postoperative cardiac rehabilitation. These refinements in perioperative practice might contribute to improvement in outcome. The current study showed that patients aged more than 80 years have about twice the risk of operative mortality of younger patients (7.3\% vs $3.1 \%)$; however, age of 80 years and older did not emerge as an independent risk factor for operative mortality. This might be due to the small number of patients in our study, but similar results have been reported in large studies analyzing outcome of AVR in octogenarians [11,14] (Table 5). Our study identified advanced NYHA class as an independent risk factor for operative mortality (Table 4); operative mortality for patients with NYHA class II was $0.7 \%$, which was significantly lower than that for those with NYHA class III-IV (8.4\%). Therefore, early referral for surgery should be important to improve surgical mortality.

\subsection{Postoperative Complications and Non-Home Discharge}

The current study showed a statistically significant increase of postoperative complications in the older patients compared with the younger patients. This is largely due to the significantly higher incidence of respiratory failure in patients aged more than 80 years. However, there was no difference in any other complication between the two age groups (Table 3). The recent literature has shown similar morbidity results, and reported that elderly patients were significantly more likely to suffer from respiratory failure than younger patients $[11,14]$.

Non-home discharge as a measure of postoperative recovery is an important issue when evaluating outcome of AVR. Our study showed that age more than 80 years was an independent predictor of non-home discharge; that the non-home discharge rate was $21.9 \%(n=9)$ in the older patients, which was 4 times higher than that in the younger patients (5.5\%). At the last follow-up in the current study, 6 out of 9 patients aged more than 80 years discharged to non-home location returned home and are leading satisfactory lives, and thus a total of $83 \%(n=34)$ 
Table 5. Operative mortality and survival for patients aged 80 years and older.

\begin{tabular}{cccccccc}
\hline & & & Patients & Operative & \multicolumn{3}{c}{ Survival (\%) } \\
\cline { 3 - 7 } Author, year & Reference & Surgery & No. & Mortality (\%) & 1-year & 3-year & 5 -year \\
\hline Florath, 2010 & 10 & AVR \pm CABG & 493 & 8.5 & 82 & NA & 52 \\
Thourani, 2008 & 11 & AVR & 88 & 5.7 & 87 & 68 & 61 \\
Chiappini, 2004 & 12 & AVR \pm CABG & 115 & 8.5 & 86 & NA & 69 \\
Melby, 2007 & 13 & AVR \pm CABG & 245 & 10 & 82 & 70 & 56 \\
Leontyev, 2009 & 15 & AVR & 282 & 10.6 & 81 & 71 & 57 \\
This study & & AVR \pm CABG & 41 & 7.3 & 89 & 84 & 84 \\
\hline
\end{tabular}

of patients aged more than 80 years $(n=41)$ who underwent AVR are living at home.

\subsection{Mid-Term Survival}

In the current study, survival estimates for patients aged more than 80 years at 1,3 , and 5 years were $89 \%, 84 \%$, and $84 \%$, respectively (Figure 1). This compares well with recent large studies reporting long-term survival of AVR in octogenarians (Table 5). Independent predictors of mid-term death identified by multivariate analysis in our study included creatinine $\geq 2 \mathrm{mg} / \mathrm{dL}$, advanced NYHA class, and peripheral vascular disease (Table 4). Age more than 80 years was not a risk factor of mid-term mortality in the current study.

\subsection{Limitations}

The current study is limited by its retrospective nature and selection bias inherent in the data. Another limitation is that relatively small number of patients, which might be a reason of why potential risk factors such as old myocardial infarction, diabetes mellitus, and concomitant $\mathrm{CABG}$, were not found to be of multivariate significance of outcomes.

\section{CONCLUSION}

Good results after AVR can be expected in patients aged 80 years and older, with slightly higher operative mortality and morbidity compared with younger patients. Midterm survival in elderly patients is very acceptable, with more than $80 \%$ survival at 3-year after AVR. More than $80 \%$ of patients are living at home with a mean followup period of 2.2 years. These satisfactory results of conventional AVR in patients aged 80 years and older, as well as independent risk factors for outcomes found in the current study have to be taken into consideration in the decision-making process regarding treatment strategy for patients with AS.

\section{REFERENCES}

[1] Iung, B., Baron, G., Butchart, E.G., et al. (2003) A pro- spective survey of patients with valvular heart disease in Europe: The Euro heart survey on valvular heart disease. European Heart Journal, 24, 1231-1243. doi:10.1016/S0195-668X(03)00201-X

[2] Vahanian, A., Baumgartner, H., Bax, J., et al. (2007) Task Force on the Management of Valvular Hearth Disease of the European Society of Cardiology; ESC Committee for Practice Guidelines. Guidelines on the management of valvular heart disease: The Task Force on the Management of Valvular Heart Disease of the European Society of Cardiology. European Heart Journal, 28, 230-268.

[3] Bonow, R.O., Carabello, B.A., Chatterjee, K., et al. (2008) 2006 Writing Committee Members; American College of Cardiology/American Heart Association Task Force. 2008 Focused update incorporated into the ACC/AHA 2006 guidelines for the management of patients with valvular heart disease: A report of the American College of Cardiology/American Heart Association Task Force on Practice Guidelines (Writing Committee to Revise the 1998 Guidelines for the Management of Patients With Valvular Heart Disease): Endorsed by the Society of Cardiovascular Anesthesiologists, Society for Cardiovascular Angiography and Interventions, and Society of Thoracic Surgeons. Circulation, 118, e523-e661. doi:10.1161/CIRCULATIONAHA.108.190748

[4] Piérard, S., Seldrum, S., De Meester, C., et al. (2011) Incidence, determinants, and prognostic impact of operative refusal or denial in octogenarians with severe aortic stenosis. Annals of Thoracic Surgery, 91, 1107-1112. doi:10.1016/j.athoracsur.2010.12.052

[5] Rodés-Cabau, J., Webb, J.G., Cheung, A., et al. (2010) Transcatheter aortic valve implantation for the treatment of severe symptomatic aortic stenosis in patients at very high or prohibitive surgical risk: Acute and late outcomes of the multicenter Canadian experience. Journal of the American College of Cardiology, 55, 1080-1090. doi:10.1016/j.jacc.2009.12.014

[6] Smith, C.R., Leon, M.B., Mack, M.J., et al. (2011) PARTNER Trial Investigators. Transcatheter versus surgical aortic-valve replacement in high-risk patients. The New England Journal of Medicine, 364, 2187-2198. doi:10.1056/NEJMoa1103510

[7] Olsson, M., Janfjäll, H., Orth-Gomér, K., Undén, A. and Rosenqvist, M. (1996) Quality of life in octogenarians after valve replacement due to aortic stenosis. A prospective comparison with younger patients. European Heart Journal, 17, 583-589. 
doi:10.1093/oxfordjournals.eurheartj.a014912

[8] Kolh, P., Lahaye, L., Gerard, P. and Limet, R. (1999) Aortic valve replacement in the octogenarians: Perioperative outcome and clinical follow-up. European Journal Cardio-Thoracic Surgery, 16, 68-73. doi:10.1016/S1010-7940(99)00146-3

[9] Deleuze, P., Loisance, D.Y., Besnainou, F. et al. (1990) Severe aortic stenosis in octogenarians: Is operation an acceptable alternative? Annals of Thoracic Surgery, 50, 226-229. doi:10.1016/0003-4975(90)90739-S

[10] Florath, I., Albert, A., Boening, A., Ennker, I.C. and Ennker, J. (2101) Aortic valve replacement in octogenarians: identification of high-risk patients. European Journal Cardio-Thoracic Surgery, 37, 1304-1310. doi:10.1016/i.ejcts.2009.12.025

[11] Thourani, V.H., Myung, R., Kilgo, P., et al. (2008) Longterm outcomes after isolated aortic valve replacement in octogenarians: A modern perspective. Annals of Thoracic Surgery, 86, 1458-1464.

\section{ABBREVIATIONS AND ACRONYMS}

$\mathrm{AS}=$ aortic valve stenosis

$\mathrm{AVR}=$ aortic valve replacement

$\mathrm{CABG}=$ coronary artery bypass grafting

$\mathrm{CI}=$ confidence interval

$\mathrm{COPD}=$ chronic obstructive pulmonary disease

$\mathrm{DM}=$ diabetes mellitus

$\mathrm{EF}=$ ejection fraction

EuroSCORE $=$ European System for Cardiac Operative doi:10.1016/j.athoracsur.2008.06.036

[12] Chiappini, B., Camurri, N., Loforte, A., et al. (2004) Outcome after aortic valve replacement in octogenarians. Annals of Thoracic Surgery, 78, 85-89. doi:10.1016/j.athoracsur.2003.12.060

[13] Melby, S.J., Zierer, A., Kaiser, S.P., et al. (2007) Aortic valve replacement in octogenarians: Risk factors for early and late mortality. Annals of Thoracic Surgery, 83, 16511656. doi:10.1016/j.athoracsur.2006.09.068

[14] Filsoufi, F., Rahmanian, P.B., Castillo, J.G., Chikwe, J., Silvay, G. and Adams, D.H. (2008) Excellent early and late outcomes of aortic valve replacement in people aged 80 and older. Journal of the American Geriatrics Society, 56, 255-261. doi:10.1111/j.1532-5415.2007.01535.x

[15] Leontyev, S., Walther, T., Borger, M.A., et al. (2009) Aortic valve replacement in octogenarians: Utility of risk stratification with EuroSCORE. Annals of Thoracic Surgery, 87, 1440-1445. doi:10.1016/j.athoracsur.2009.01.057

Risk Evaluation

NYHA $=$ New York Heart Association

$\mathrm{OR}=$ odds ratio

$\mathrm{O} / \mathrm{E}=$ observed/expected

PLOS = prolonged length of stay

STS-PROM = Society of Thoracic Surgeons Predicted

Risk of Mortality

TAVI $=$ transcatheter aortic valve implantation 